\title{
CURRENT TOPICS IN COMPLEMENT
}




\section{ADVANCES IN EXPERIMENTAL MEDICINE AND BIOLOGY}

Editorial Board:

NATHAN BACK, State University of New York at Buffalo

IRUN R. COHEN, The Weizmann Institute of Science

DAVID KRITCHEVSKY, Wistar Institute

ABEL LAJTHA, N.S. Kline Institute for Psychiatric Research

RODOLFO PAOLETTI, University of Milan

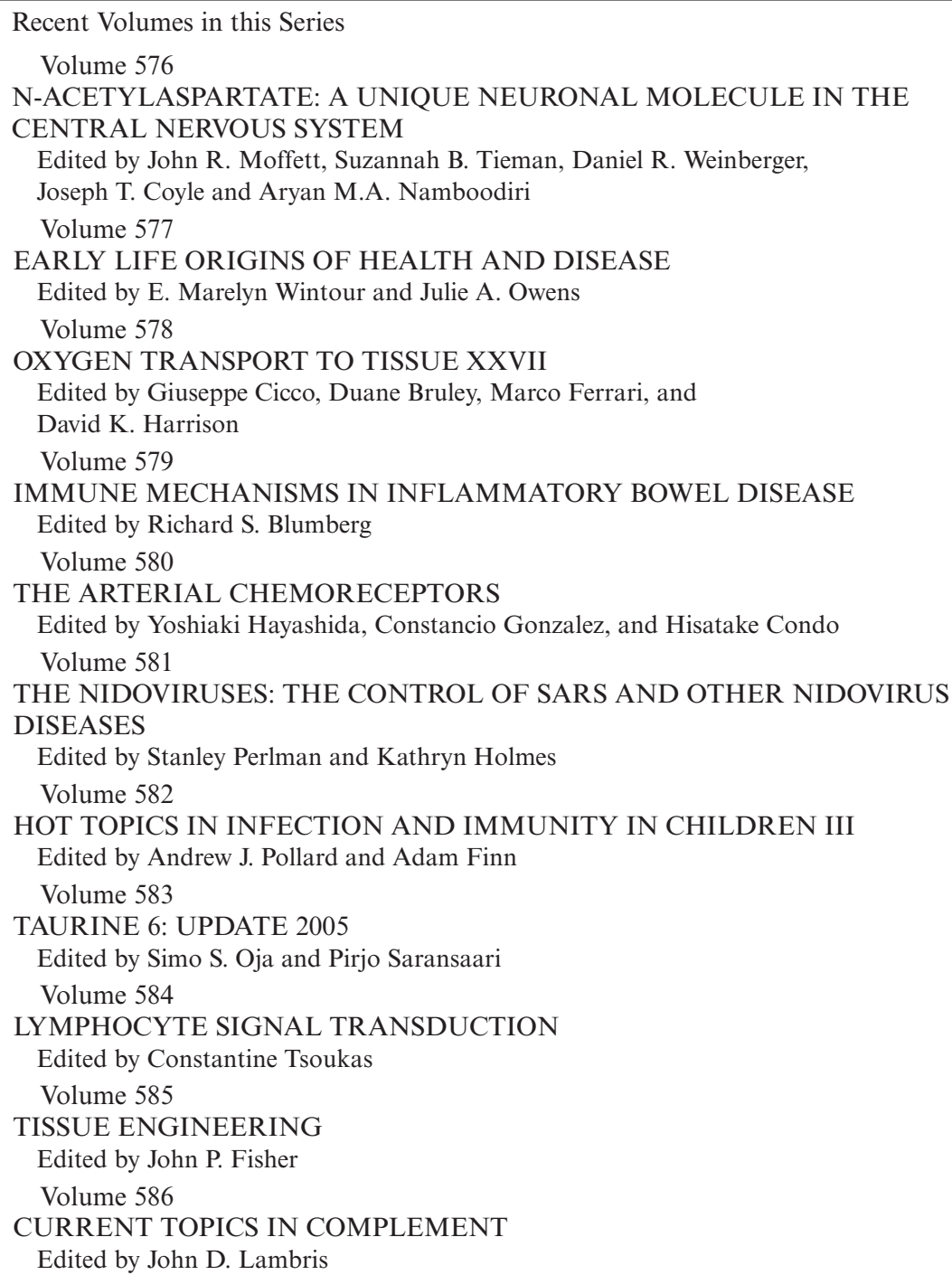

Recent Volumes in this Series

Volume 576

N-ACETYLASPARTATE: A UNIQUE NEURONAL MOLECULE IN THE

CENTRAL NERVOUS SYSTEM

Edited by John R. Moffett, Suzannah B. Tieman, Daniel R. Weinberger,

Joseph T. Coyle and Aryan M.A. Namboodiri

Volume 577

EARLY LIFE ORIGINS OF HEALTH AND DISEASE

Edited by E. Marelyn Wintour and Julie A. Owens

Volume 578

OXYGEN TRANSPORT TO TISSUE XXVII

Edited by Giuseppe Cicco, Duane Bruley, Marco Ferrari, and

David K. Harrison

Volume 579

IMMUNE MECHANISMS IN INFLAMMATORY BOWEL DISEASE

Edited by Richard S. Blumberg

Volume 580

THE ARTERIAL CHEMORECEPTORS

Edited by Yoshiaki Hayashida, Constancio Gonzalez, and Hisatake Condo

Volume 581

THE NIDOVIRUSES: THE CONTROL OF SARS AND OTHER NIDOVIRUS

DISEASES

Edited by Stanley Perlman and Kathryn Holmes

Volume 582

HOT TOPICS IN INFECTION AND IMMUNITY IN CHILDREN III

Edited by Andrew J. Pollard and Adam Finn

Volume 583

TAURINE 6: UPDATE 2005

Edited by Simo S. Oja and Pirjo Saransaari

Volume 584

LYMPHOCYTE SIGNAL TRANSDUCTION

Edited by Constantine Tsoukas

Volume 585

TISSUE ENGINEERING

Edited by John P. Fisher

Volume 586

CURRENT TOPICS IN COMPLEMENT

Edited by John D. Lambris

A Continuation Order Plan is available for this series. A continuation order will bring delivery of each new volume immediately upon publication. Volumes are billed only upon actual shipment. For further information please contact the publisher. 


\section{CURRENT TOPICS IN COMPLEMENT}

Edited by

John D. Lambris

鱼 Springer 


\section{Editor:}

John D. Lambris, Ph.D.

Professor of Pathology \& Laboratory Medicine

University of Pennsylvania

Philadelphia, PA 19107

lambris@mail.med.upenn.edu

Library of Congress Control Number: 2006925169

Printed on acid-free paper.

ISBN 10: 0-387-32231-0

ISBN-13: 978-0387-32231-5

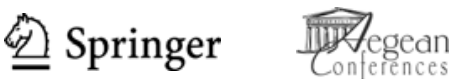

(C) 2006 Springer Science+Business Media, LLC

All rights reserved. This work may not be translated or copied in whole or in part without the written permission of the publisher (Springer Science+Business Media, LLC, 233 Spring Street, New York, NY 10013, USA), except for brief excerpts in connection with reviews or scholarly analysis. Use in connection with any form of information storage and retrieval, electronic adaptation, computer software, or by similar or dissimilar methodology now known or hereafter developed is forbidden.

The use in this publication of trade names, trademarks, service marks and similar terms, even if they are not identified as such, is not to be taken as an expression of opinion as to whether or not they are subject to proprietary rights.

Printed in the United States of America.

988765543221

springer.com 


\section{Preface}

Complement has long been regarded as a pivotal effector arm of the innate immune response, eliciting important immunoregulatory functions in the context of inflammation and also serving as a vital link between the innate and adaptive immune response. In the post-genomic era, our knowledge of the innate immune system is enriched by findings that point to novel functions that do not strictly correlate with immunological defense and surveillance, immune modulation or inflammation. Several studies indicate that complement proteins exert functions that are either more complex than previously thought, or go well beyond the innate immune character of the system.

The advent of high-throughput platforms for genome and proteome-wide profiling, together with the enormous amount of raw genetic information that has accumulated in the databases, have stirred new expectations in biomedical research. They have led complementologists to revisit established biological systems, such as the complement system, from a global and integrative perspective. Complement research is now faced with the challenge of trying to integrate isolated biochemical pathways into complex gene and protein regulatory circuits. In this respect, scientists from around the world convened at the Third Aegean Conferences Workshop on Complement Associated Diseases, Animal Models, and Therapeutics (June 5-10, 2005), to discuss recent advances in this fast evolving field. This volume represents a collection of topics on the "novel" functions of complement, pathophysiology, protein structures, design of complement inhibitors, and complement assays discussed during the conference.

I am grateful to the contributing authors for the time and effort they have devoted to writing, what I consider exceptionally informative chapters in a book that will have a significant impact on the complement field. I am grateful to Rodanthi Lambris for her assistance in formatting the text. I also gratefully acknowledge the generous help provided by Dimitrios Lambris in managing the organization of this meeting. Finally, I also thank Andrea Macaluso of Springer Publishers for her supervision in this book's production.

John D. Lambris, PhD 


\section{Contents}

List of Contributors

xvii

1. Cross-Disciplinary Research Stirs New Challenges into the Study of the Structure, Function and Systems Biology of Complement

Dimitrios Mastellos and John D. Lambris

1. Introduction

2. Biophysical Approaches in Elucidating Complement Structure and Binding Energetics

3. Thermodynamics of Complement Protein Binding...

4. Probing Conformational Changes of Complement Proteins with Hydrogen/Deuterium Exchange and Mass Spectrometry...

5. Combinatorial and in Silico Protein Design: In Search for More Potent C3 Inhibitors

6. Defining the Structural Determinants of Viral Immune Evasion: The C3b/SPICE/VCP Interaction

7. A "Systems Biology" Perspective of Innate Immunity:

Newly Identified "Crosstalks" between Complement and

Divergent Biological Networks

7.1. Complement Intercepts Cytokine-Driven Regenerative Networks in the Liver

7.2. A Complement-Chemokine "Crosstalk" Regulates

Hematopoietic Stem Cell Engraftment

7.3. Complement Modulates Coagulation Processes ............................... $\quad 10$

8. Future Perspectives ............................................................................... 11

9. Acknowledgments.......................................................................... 12

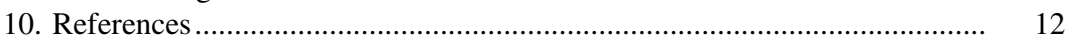

2. Liver Regeneration: A Link to Inflammation through Complement

Robert A. DeAngelis, Maciej M. Markiewski, and John D. Lambris

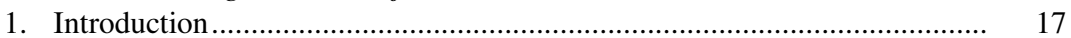

2. Liver Regeneration and Inflammatory Mediators ..................................... 18

2.1. Cytokines and Transcription Factors ............................................. 19

2.2. Growth Factors, Metalloproteases, Adhesion Molecules, and Acute Phase Proteins

2.3. Natural Killer T (NKT) Cells............................................................ 22

3. The Role of Complement in Liver Regeneration.......................................... 23

4. Conclusion ........................................................................................... 23

5. Acknowledgments..................................................................... 26

6. References ............................................................................. 26 
3. The Role of Third Complement Component (C3) in Homing of Hematopoietic Stem/Progenitor Cells into Bone Marrow Ryan Reca, Marcin Wysoczynski, Jun Yan, John D. Lambris, and Mariusz Z. Ratajczak

1. Introduction

2. The Function of CXCR4 Receptor Depends on Lipid Raft Formation........ 36

3. Complement Is Activated in BM during Myeloablative Conditioning for Hematopoietic Transplantation.

4. The Role of Complement in Regulating the Biology of HSPC ................... 39

5. Hematopoiesis in C3-Deficient Mice under Normal Steady-State and Stress Situations

6. Molecular Explanation of the Defect in Homing/Engraftment of HSPC in C3-Deficient Mice

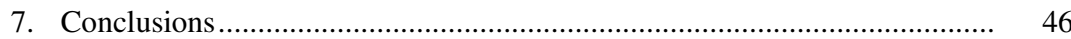

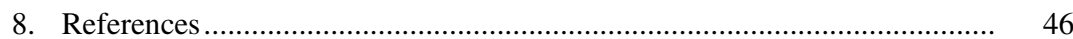

4. Complement System and the Eye

Purushottam Jha, Puran S. Bora, Jeong-Hyeon Sohn,

Henry J. Kaplan, and Nalini S. Bora

1. Introduction

2. Complement and Ocular Protection ........................................................ 54

3. Complement and Ocular Diseases................................................................ 54

3.1. Complement and Corneal Diseases.................................................. 54

3.2. Complement and Autoimmune Uveitis.............................................. 55

3.3. Complement and Age-Related Macular Degeneration...................... 55

4. Complement and Ocular Tolerance ............................................................ 56

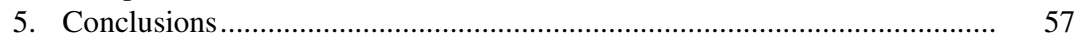

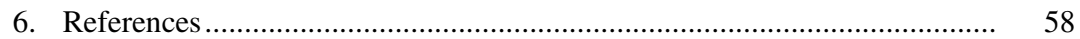

\section{To Regeneration ... With Complement}

Panagiotis A. Tsonis, John D. Lambris, and Katia Del Rio-Tsonis

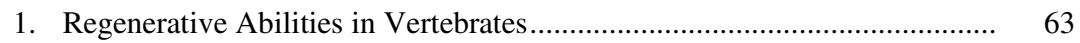

2. Limb Regeneration..................................................................... 64

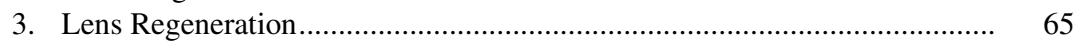

4. The Complement System ................................................................... 66

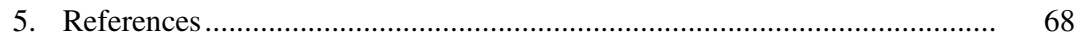

\section{Self, Non-Self and Danger: A Complementary View} Jörg Köhl

1. Introduction

2. Complement as a "Master Alarm System" of Innate Immunity .................. 72

3. Complement-Derived Danger-Transmitters Shape Innate and Adaptive Immune Responses following Physiological and Pathological Threats

3.1. Danger Transmission through C1q Receptors ................................. 75

3.2. Danger Transmission through C3 Cleavage Fragments..................... 75 
4. Danger Transmission Mediated through the Anaphylatoxic

Peptides C3a and C5a......................................................................... 78

4.1. Anaphylatoxin Receptor-Dependent and -Independent Effects .......... 79

5. Anaphylatoxin-Mediated Danger Transmission in Non-Myeloid Cells ....... 81

6. C5a Receptor Signaling in Pulmonary Dendritic Cells Regulates Inhalation Tolerance.

7. C5a Receptor Signaling on APCs Impacts Danger Transmission through TLRs

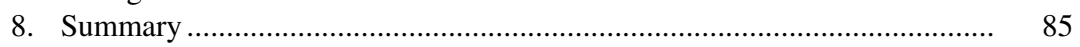

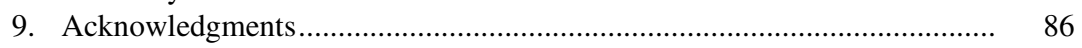

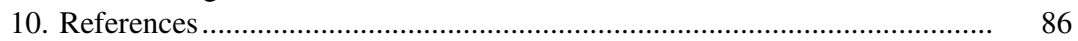

7. $\mathrm{gC1qR/p33} \mathrm{Serves} \mathrm{as} \mathrm{a} \mathrm{Molecular} \mathrm{Bridge} \mathrm{between} \mathrm{the}$ Complement and Contact Activation Systems and Is an Important Catalyst in Inflammation

Berhane Ghebrehiwet, Claudia Cebada-Mora, Lee Tantral, Jolyon Jesty, and Ellinor I. B. Peerschke

1. Abstract.....

2. Introduction …..................................................................................... 96

3. Materials and Methods ..................................................................... 97

3.1. Chemicals and Reagents …….................................................... 97

3.2. Proteins and Antibodies .................................................................. 97

3.3. Biotinylation of Proteins .................................................................. 97

3.4. Expression of Recombinant gC1qR ................................................. 98

3.5. Collection of Normal Human Serum ............................................ 98

3.6. Hemolytic Assay ......................................................................... 98

3.7. Microplate Assay for Complement Activation ................................. 99

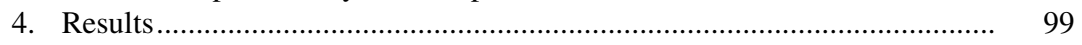

4.1. Inhibition of Hemolytic Activity by gC1qR ................................... 99

4.2. Soluble $\mathrm{gC} 1 \mathrm{qR}$ but not $\Delta 74-96 \mathrm{gClqR}$ Can Activate the Classical Pathway .................................................................. 100

5. Discussion .......................................................................................... 101

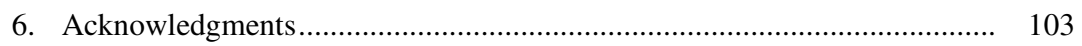

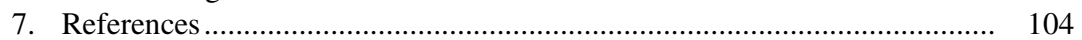

8. Possible Immunoprotective and Angiogenesis-Promoting Roles for Malignant Cell-Derived Prostasomes: A New Paradigm for Prostatic Cancer?

Kristina Nilsson Ekdahl, Gunnar Ronquist, Bo Nilsson, and Adil A. Babiker

1. Introduction

2. Hypothesis: Malignant Cell-Derived Prostasomes Provide Cancer Cells with a Zone of Innate Immune Privilege.

3. Complement Activation and Expression of Complement Regulatory Proteins by Malignant Cells

4. CD59 Transfer by Prostasomes Results in Protection against Complement-Mediated Lysis

5. Extracellular Phosphorylation of Plasma Proteins. 
6. Mapping of Protein Kinases on Prostasomes ........................................... 114

7. C3 and other Substrates for Prostasomal PKs .......................................... 115

8. Conclusions ........................................................................................ 116

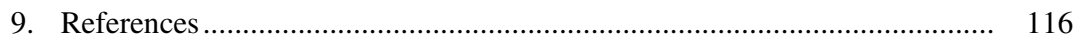

9. Diversified Components of the Bony Fish Complement System: More Genes for Robuster Innate Defense?

Miki Nakao, Yoko Kato-Unoki, Makiko Nakahara, Junichi Mutsuro, and Tomonori Somamoto

1. Introduction

2. Classical Pathway Components............................................................. 123

3. Lectin Pathway Components............................................................... 125

4. Alternative Pathway Components ........................................................... 126

5. Lytic Pathway Components................................................................ 131

6. Complement Receptors .................................................................................. 131

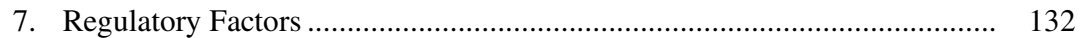

8. Concluding Remarks and Future Directions............................................... 133

9. Acknowledgments.............................................................................. 134

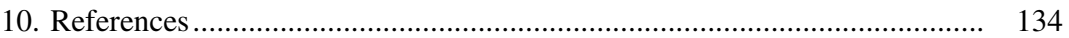

10. C5b-9 Complement Complex in Autoimmune Demyelination: Dual Role in Neuroinflammation and Neuroprotection

Horea Rus, Cornelia Cudrici, and Florin Niculescu

1. Introduction ................................................................................ 139

2. Role of C5b-9 in Neuroinflammation.................................................... 140

2.1. In Vitro Demyelination by C5b-9 ........................................... 140

2.2. Role of C5b-9 in Demyelination during EAE..................................... 141

3. Role of C5b-9 in Neuroprotection ........................................................ 142

3.1. Inhibition of Oligodendrocyte Apoptosis by Sublytic C5b-9........... 142

3.2. Contribution of Complement C5 to Neuroprotection in EAE............ 145

4. Does C5b-9 Protect Oligodendrocytes from Apoptosis in

Multiple Sclerosis?..................................................................... 146

5. Acknowledgments.................................................................. 148

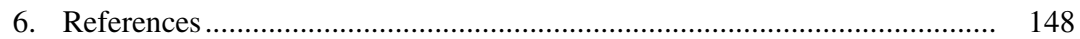

11. The Double-Edged Flower: Roles of Complement Protein C1q in Neurodegenerative Diseases

Andrea J. Tenner and Maria I. Fonseca

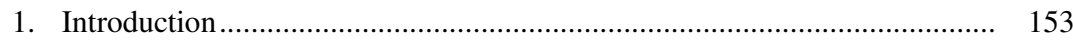

2. Complement in the Brain ................................................................ 155

3. Murine Models of Alzheimer's Disease.................................................... 157

4. Potential Protective Roles of Complement in the CNS ............................. 162

5. Potential Complement-Based Therapeutics.............................................. 165

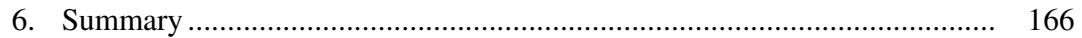

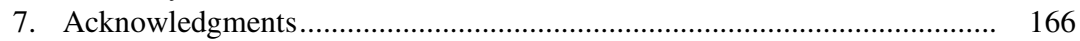

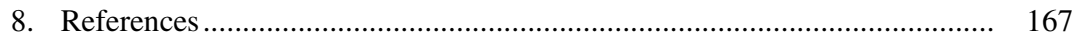


12. The Role of the Complement System in the Pathogenesis of Experimental Autoimmune Encephalomyelitis and Multiple Sclerosis

Nóra Terényi, József Prechl, and Anna Erdei

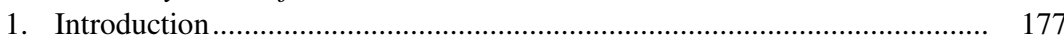

2. Local Production as a Complement Source in the CNS ........................... 180

3. The Role of Complement Deposition in Myelin Damage .......................... 180

3.1. Decomplementation by CVF ........................................................... 180

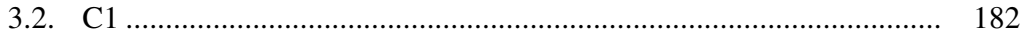

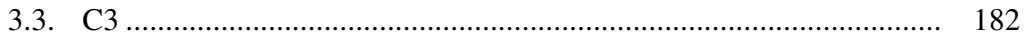

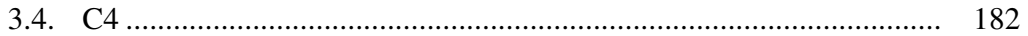

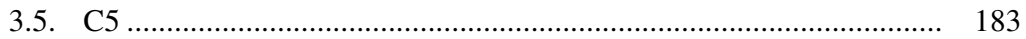

3.6. C6-C9, MAC ……................................................................. 183

4. Anaphylatoxin Effects in Demyelinization .............................................. 183

5. Complement Regulation in the CNS .................................................... 185

6. Complement and Therapy of EAE ……..................................................... 185

7. Acknowledgments................................................................ 186

8. References ............................................................................. 186

13. The Complement System: A Potential Target for Stroke Therapy

J. Mocco, Michael E. Sughrue, Andrew F. Ducruet, Ricardo

J. Komotar, Sergei A. Sosunov, and E. Sander Connolly Jr.

1. Introduction

2. Rationale for Blocking Complement Activation to Treat Stroke.................. 190

2.1. Inflammation Is Deleterious in Stroke, and Complement Is Activated in Stroke

2.2. Complement Activation Exacerbates Ischemic Injury in Other Organs

2.3. Complement Activation Causes Injury in Other Nervous System Diseases

2.4. Neurons Seem to Be Unusually Susceptible to Complement Activation.

2.5. In Vivo Evidence Suggests that Complement Is Involved in Cerebral I/R Pathogenesis.

3. Potential Negatives of Complement Blockade following Stroke ................ 193

3.1. Complement May Be Needed to Opsonize Cellular Debris after Stroke

3.2. Complement Aids Tissue Recovery/Repair in Other Organs............. 194

3.3. Complement Activation Products May Be Neuroprotective............... 195

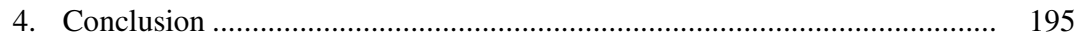

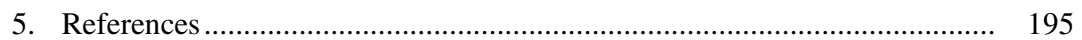

14. Observations on Complement Activity in the Two-Stage Inflammatory/Hemostatic Response in the Baboon and Human Models of $E$. Coli Sepsis and Endotoxemia Fletcher B. Taylor Jr., Eric Hack, and Florea Lupu

1. Introduction 
2. Description of the Baboon and Human Models of E. Coli Sepsis and Endotoxemia.

2.1. Baboon E. Coli Sepsis Model ............................................................ 204

2.2. Human Endotoxin Model.................................................................. 204

3. Results................................................................................... 206

3.1. Activation Parameters of the Complement System in Baboons after Lethal and Sublethal E. Coli Challenge ....................... 206

3.2. Activation Parameters of Cytokine Complement and Hemostatic Systems in Humans after Endotoxin Challenge: Evidence Establishing Two Distinct Sequential Pathophysiologic Events

3.3. Evidence Suggesting that There Is a Unique Counterpart to the Second Stage of the Compensated Response to E. Coli that Is Distinct from the Lethal Counterpart to the First Stage.

4. Conclusions .............................................................................. 210

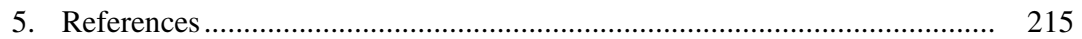

\section{Complement Activation during Sepsis in Humans}

Heike Schreiber, Daniel Rittirsch, Michael Flierl, Uwe Brueckner, Marion Schneider, Manfred Weiss, Florian Gebhard, and Markus Huber-Lang

1. Introduction

2. Material and Methods............................................................................... 218

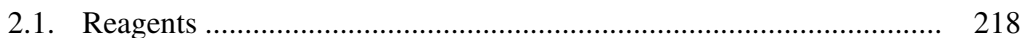

2.2. Patient Selection ............................................................... 218

2.3. Measurement of Serum Concentrations of

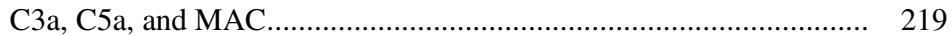

2.4. Hemolytic Complement Assay ...................................................... 219

2.5. Neutrophil Isolation ................................................................. 219

2.6. Analysis of C5aR Content on Neutrophils..................................... 219

2.7. Statistical Analysis................................................................ 220

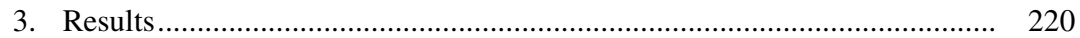

3.1. Epidemiological Assessments........................................................ 220

3.2. Sepsis-Induced Complement Activation during

3.3. Sepsis-Induced Impairment of Complement Function during Septic Shock in Humans....................................................... 221

3.4. Loss of C5aR on Neutrophils Is Associated with a
Lethal Outcome during Sepsis in Humans ....................................... 221

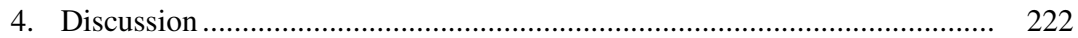

5. Acknowledgments............................................................................ 224

6. References .......................................................................... 225 
16. Three Distinct Profiles of Serum Complement C4 Proteins in Pediatric Systemic Lupus Erythematosus (SLE) Patients: Tight Associations of Complement C4 and C3 Protein Levels in SLE but not in Healthy Subjects

Yee-Ling Wu, Gloria C. Higgins, Robert M. Rennebohm, Erwin K. Chung, Yan Yang, Bi Zhou, Haikady N. Nagaraja,

Dan J. Birmingham, Brad H. Rovin, Lee A. Hebert, and C. Yung Yu

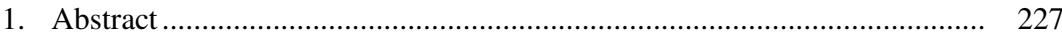

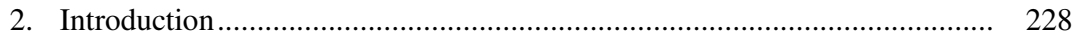

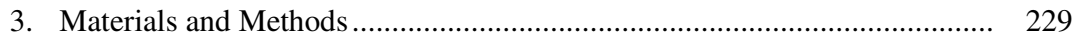

3.1. Study Populations .................................................................... 229

3.2. Preparation of EDTA-Plasma and Genomic DNAs ......................... 229

3.3. C4 Phenotyping and Genotyping ................................................. 229

3.4. Mutations of Complement $C 4$ and $C 2$ Genes................................ 230

3.5. Clinical Information...................................................................... 230

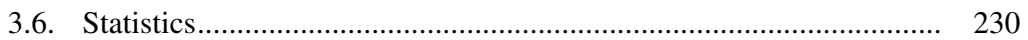

4. Results ......................................................................................... 230

4.1. Demographics and Clinical Features of the Pediatric

SLE Study Population........................................................................ 231

4.2. Three Types of C3-C4 Protein Profiles in SLE Patients ................... 233

4.3. Tight Correlation between Serum $\mathrm{C} 3$ and $\mathrm{C} 4$ Concentrations in SLE Patients but not in Healthy Subjects

4.4. Serum C3 and C4 Levels Both Correlated BMI but BMI Alone Could not Account for the Tight Association between Serum C3 and C4 Levels .............................. 238

4.5. C4 Genotypic and Phenotypic Variations in Pediatric SLE............... 239

4.6. $C 4$ Gene Dosage Is a Determinant of the Maximum Serum C4 Concentrations in Pediatric SLE ...................................... 241

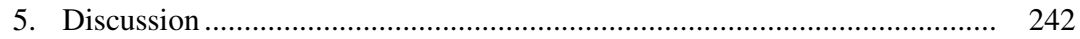

6. Acknowledgments.............................................................................. 244

7. References ........................................................................... 244

17. A Minimum CR2 Binding Domain of C3d Enhances Immunity following Vaccination

Joseph F. Bower and Ted M. Ross

1. Abstract .......................................................................................... 249

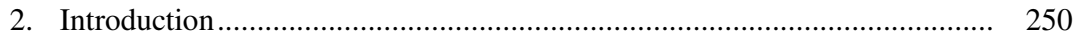

3. Materials and Methods …................................................................ 251

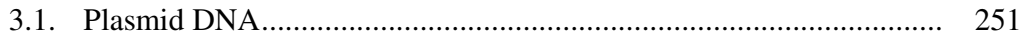

3.2. Purification of Recombinant Protein Antigens ............................... 251

3.3. Protein Expression ............................................................. 252

3.4. Animals and Immunizations ..................................................... 252

3.5. ELISA ......................................................................... 253

3.6. ELISpot.............................................................................. 253

3.7. Statistical Analysis.................................................................. 254

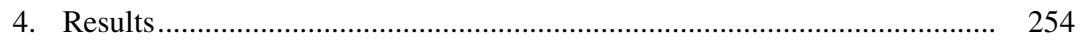

4.1. Expression of Vaccine Plasmids ................................................. 254 
4.2. Cell-Mediated Immune Responses Elicited by Env-mC3d $\ldots \ldots \ldots \ldots \ldots . .255$

4.3. Anti-Env Antibody Responses................................................... 256

4.4. Mucosal Immunizations...................................................................... 256

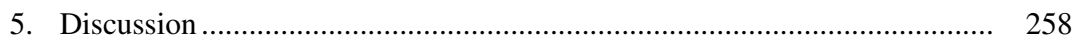

6. Acknowledgments..................................................................... 260

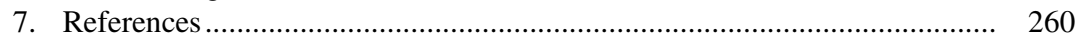

\section{Structure and Function of Ficolins}

Yuichi Endo, Yu Liu, and Teizo Fujita

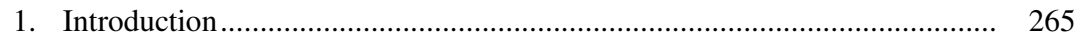

2. Structure of Ficolin ................................................................................ 266

3. Tissue and Cell Type Expressing Ficolin ................................................. 268

4. Phylogeny of the Ficolin Family .............................................................. 269

5. Function of Ficolin ................................................................................ 271

5.1. Carbohydrate Binding of Ficolin ............................................... 271

5.2. Binding of Ficolin to Bacteria....................................................... 271

5.3. Ficolin as a Recognition Molecule in the Lectin Pathway ................. 274

6. Polymorphisms of the Ficolin Gene ……................................................. 275

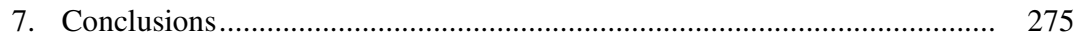

8. Acknowledgments........................................................................ 276

9. References ............................................................................. 276

19. Role of Mannose-Binding Lectin (MBL2) Genotyping in Predicting the Risk of Recurrent Otitis Media (rOM) Lieve Nuytinck, Els De Meester, Martine Van Thielen, and Paul Govaerts

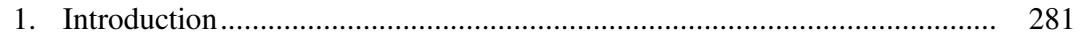

2. MBL2 Gene and Polymorphisms............................................................. 283

3. Materials and Methods ................................................................... 284

3.1. Patients and Controls .............................................................. 284

3.2. MBL2 Genotyping …........................................................... 285

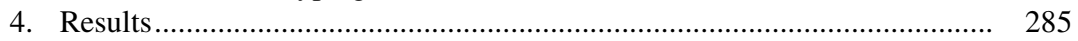

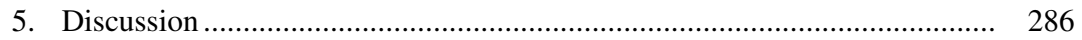

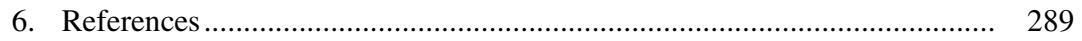

20. Conformational Complexity of Complement Component C3

Bert J.C. Janssen and Piet Gros

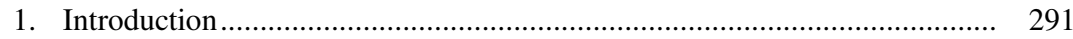

2. Structural Organization of C3 ….................................................... 292

3. Convertase Formation ......................................................................... 296

4. Decay Acceleration ............................................................................ 299

5. Cofactor Activity .................................................................................... 301

6. Signaling Roles of C3B Fragments ................................................... 302

7. Concluding Remarks .................................................................... 303

8. Acknowledgments............................................................................ 304

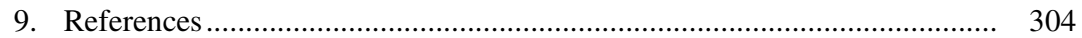


21. Disease-Associated Sequence Variations in Factor $\mathbf{H}$ :

A Structural Biology Approach

Andrew P. Herbert, Dinesh C. Soares, Michael K. Pangburn, and Paul N. Barlow

1. Introduction

2. Regulation of the Complement System .................................................. 314

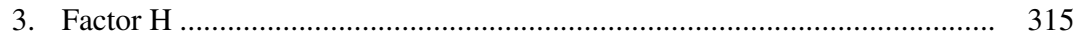

4. Functional Sites of Factor H............................................................... 317

5. Atypical Hemolytic Uremic Syndrome ................................................. 318

6. Age-Related Macular Degeneration .................................................... 318

7. Modeled Modules of Factor H .............................................................. 319

8. Predicted Structural Consequences of Amino-Acid Substitutions............... 321

9. References ......................................................................................... 323

22. Transdermal Pharmacology of Small Molecule Cyclic C5a Antagonists

Lavinia M. Proctor, Trent M. Woodruff, Prakirti Sharma, Ian A. Shiels, and Stephen M. Taylor

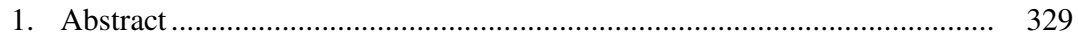

2. Introduction ........................................................................... 330

3. Material and Methods...................................................................... 332

3.1. Materials .................................................................................. 332

3.2. Isolation of Polymorphonuclear Leukocytes..................................... 332

3.3. Receptor Binding Assay .......................................................... 333

3.4 Myeloperoxidase Release from PMNs.......................................... 333

3.5. In Vivo Studies ..................................................................... 333

3.6. Statistical Analysis............................................................... 334

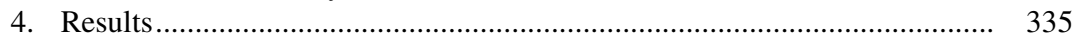

4.1. In Vitro Activity of PMX Compounds........................................... 335

4.2. Transdermal Pharmacokinetics of Cyclic C5a Receptor Antagonists................................................................. 335

4.3. Effect of Administration of C5a Antagonists on

LPS-Induced Neutropenia and Hypotension.................................. 337

5. Discussion ............................................................................. 341

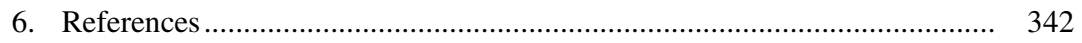

23. Inactivation of Complement by Recombinant Human C3 Derivatives

Edzard Spillner, Johanna Kölln, and Reinhard Bredehorst

1. Introduction ...................................................................................... 347

2. Generation of CVF Chimeras and C3 Derivatives ................................... 349

3. Functional Characteristics of the C3 Derivatives ....................................... 351

4. The C345C Domain in Complement ...................................................... 353

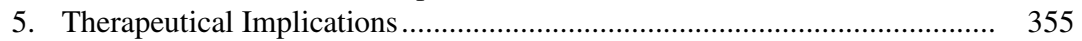

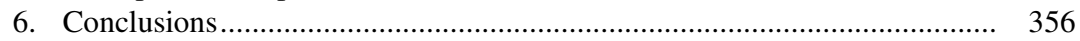

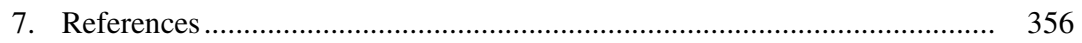




\section{Complement Analysis in Clinic and Research}

Tom E. Mollnes and Michael Kirschfink

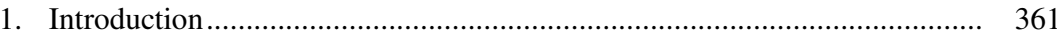

1.1. The Complement System ............................................................ 361

2. Clinical Indications for Complement Analysis......................................... 363

2.1. Recurrent Infections.............................................................. 364

2.2. Autoimmune Diseases ............................................................... 365

2.3. Membranoproliferative Glomerulonephritis (MPGN) and Hemolytic Uremic Syndrome (HUS) ........................................ 365

2.4. Hereditary Angioedema ................................................................ 366

2.5. Paroxysmal Nocturnal Hemoglobinuria (PNH) ............................. 366

3. Complement Tests................................................................ 366

3.1. Functional Assays .................................................................... 366

3.2. Protein Quantification of Individual Components ............................ 370

3.3. Genetic Analysis ................................................................... 370

3.4. Cell Surface Expression of Complement Proteins ............................. 370

3.5. Analysis of Complement Activation Products ................................. 370

4. Complement Analysis in Experimental Settings ......................................... 372

4.1. In Vitro Experiments with Human Serum and Blood ...................... 372

4.2. Animal Experiments .................................................................. 373

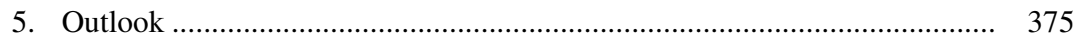

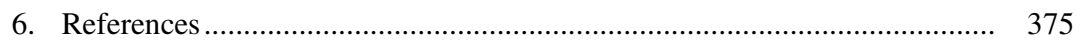

25. Cell-Bound Complement Activation Products (CB-CAPs) as a Source of Lupus Biomarkers

Sarah J. Calano, Pei-an B. Shih, Chau-Ching Liu, Amy H. Kao, Jeannine S. Navratil, Susan Manzi, and Joseph M. Ahearn

1. Introduction ..................................................................................... 381

2. Measurement of Complement in SLE ................................................... 382

2.1. Serum C3 and C4 and SLE Disease Activity ............................... 382

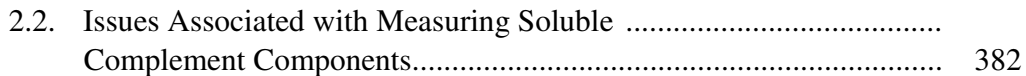

2.3. Complement Activation Products and SLE Disease Activity ............ 384

3. Cell-Bound Complement Activation Products .......................................... 384

3.1. Erythrocyte-Bound C4d as a Diagnostic Assay for SLE................... 385

3.2. Reticulocyte-Bound C4d as an "Instant Messenger" of Disease Activity in SLE............................................................... 387

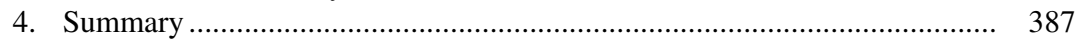

5. Acknowledgments...................................................................... 388

6. References ..................................................................................... 388

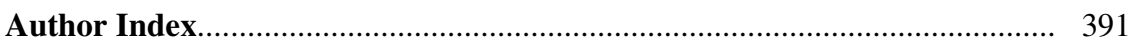

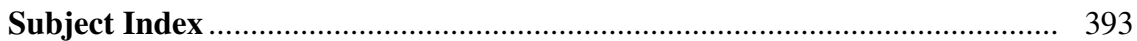




\section{List of Contributors}

Joseph M. Ahearn

Lupus Center of Excellence

University of Pittsburgh School

of Medicine

Pittsburgh, Pennsylvania, USA

Adil A. Babiker

Department of Oncology, Radiology, and Clinical Immunology

University Hospital

Uppsala, Sweden

Paul N. Barlow

Joseph Black Chemistry Building

University of Edinburgh

Edinburgh, UK

Dan J. Birmingham

Department of Internal Medicine

The Ohio State University

Columbus, Ohio, USA

Nalini S. Bora

Department of Ophthalmology

Jones Eye Institute

University of Arkansas for Medical Sciences

Little Rock, Arkansas, USA

Puran S. Bora

Department of Ophthalmology

Jones Eye Institute

University of Arkansas for

Medical Sciences

Little Rock, Arkansas, USA

Joseph F. Bower

Department of Medicine

Division of Infectious Diseases

University of Pittsburgh

Pittsburgh, Pennsylvania, USA
Reinhard Bredehorst

Institut für Biochemie und Lebensmittelchemie

Universität Hamburg, Germany

Uwe Brueckner

Department of Traumatology, Hand-

Plastic and Reconstructive Surgery

Ulm, Germany

Sarah J. Calano

Department of Medicine

University of Pittsburgh

Pittsburgh, Pennsylvania, USA

Claudia Cebada-Mora

Department of Medicine

SUNY at Stony Brook

Stony Brook, New York, USA

Erwin K. Chung

Columbus Children's Research Institute

The Ohio State University

Columbus, Ohio, USA

E. Sander Connolly Jr.

Department of Neurological Surgery

Columbia University

College of Physicians \& Surgeons

New York, New York, USA

Cornelia Cudrici

Department of Neurology

University of Maryland

School of Medicine

Baltimore, Maryland, USA

Robert A. DeAngelis

Department of Pathology and Laboratory Medicine

University of Pennsylvania

Philadelphia, Pennsylvania, USA 
Katia Del Rio-Tsonis

Department of Zoology

Miami University

Oxford, Ohio, USA

Els De Meester

Innogenetics NV

Ghent, Belgium

Andrew F. Ducruet

Department of Neurological Surgery

Columbia University

College of Physicians \& Surgeons

New York, New York, USA

Yuichi Endo

Department of Immunology

Fukushima Medical University

School of Medicine

Fukushima, Japan

Anna Erdei

Department of Immunology

Eötvös Loránd University

Budapest, Hungary

Micheal Flierl

Department of Traumatology, Hand-

Plastic and Reconstructive Surgery

Ulm, Germany

Maria I. Fonseca

Departments of Molecular Biology and Biochemistry and Pathology

University of California

Irvine, California, USA

Teizo Fujita

Department of Immunology

Fukushima Medical University

School of Medicine

Fukushima, Japan

Florian Gebhard

Department of Traumatology,

Hand-Plastic and

Reconstructive Surgery

Ulm, Germany
Berhane Ghebrehiwet

Department of Medicine

SUNY at Stony Brook

Stony Brook, New York, USA

Paul Govaerts

The Eargroup

Antwerpen-Deurne, Belgium

Piet Gros

Department of Chemistry

Utrecht University

Utrecht, The Netherlands

Eric Hack

Department of Immunopathology

Sanquin Research

Amsterdam, The Netherlands

Lee A. Hebert

Department of Internal Medicine

The Ohio State University

Columbus, Ohio, USA

Andrew P. Herbert

School of Chemistry/Institute of Structural and Molecular Biology

University of Edinburgh

Edinburgh, UK

Gloria C. Higgins

Columbus Children's Research Institute

Department of Pediatrics

The Ohio State University

Columbus, Ohio, USA

Markus Huber-Lang

Department of Traumatology, HandPlastic and Reconstructive Surgery

Ulm, Germany

Bert J.C. Janssen

Department of Chemistry

Utrecht University

Utrecht, The Netherlands 
Jolyon Jesty

Department of Medicine

SUNY at Stony Brook

Stony Brook, New York, USA

Purushottam Jha

Department of Ophthalmology

Jones Eye Institute

University of Arkansas for

Medical Sciences

Little Rock, Arkansas, USA

Amy H. Kao

Department of Medicine

University of Pittsburgh

Pittsburgh, Pennsylvania, USA

Henry J. Kaplan

Department of Ophthalmology and

Visual Science

University of Louisville

Louisville, Kentucky, USA

Yoko Kato-Unoki

Department of Bioscience and

Biotechnology

Kyushu University

Hakozaki, Fukuoka, Japan

Michael Kirschfink

Institute of Immunology

University of Heidelberg

Heidelberg, Germany

Jörg Köhl

Division of Molecular Immunology

Cincinnati Children's Hospital

Research Foundation

Cincinnati, Ohio, USA

Johanna Kölln

Institut für Biochemie und

Lebensmittelchemie

Universität Hamburg, Germany

Ricardo J. Komotar

Department of Neurological

Surgery

Columbia University

New York, New York, USA
John D. Lambris

Department of Pathology and Laboratory Medicine

University of Pennsylvania

Philadelphia, Pennsylvania, USA

Chau-Ching Liu

Department of Medicine

University of Pittsburgh

Pittsburgh, Pennsylvania, USA

Yu Liu

Department of Immunology

Fukushima Medical University

School of Medicine

Fukushima, Japan

Florea Lupu

Oklahoma Medical Research

Foundation

Oklahoma City, Oklahoma, USA

Susan Manzi

Department of Medicine

University of Pittsburgh

Pittsburgh, Pennsylvania, USA

Maciej M. Markiewski

Department of Pathology and Laboratory Medicine

University of Pennsylvania

Philadelphia, Pennsylvania, USA

Dimitrios Mastellos

National Center for Scientific Research "Demokritos"

Athens, Greece

J. Mocco

Department of Neurological Surgery

Columbia University

College of Physicians \& Surgeons

New York, New York, USA

Tom E. Mollnes

Institute of Immunology

Rikshospitalet University Hospital

University of Oslo, Norway 
Junichi Mutsuro

Department of Bioscience and

Biotechnology

Kyushu University

Hakozaki, Fukuoka, Japan

Haikady N. Nagaraja

Department of Statistics

The Ohio State University

Columbus, Ohio, USA

Makiko Nakahara

Department of Bioscience and Biotechnology

Kyushu University

Hakozaki, Fukuoka, Japan

Miki Nakao

Department of Bioscience and Biotechnology

Kyushu University

Hakozaki, Fukuoka, Japan

Jeannine S. Navratil

Department of Medicine

University of Pittsburgh

Pittsburgh, Pennsylvania, USA

Florin Niculescu

Department of Medicine

University of Maryland

Baltimore, Maryland, USA

Bo Nilsson

Department of Medical Sciences

Uppsala University Hospital

Uppsala, Sweden

Kristina Nilsson-Ekdahl

Department of Chemistry and

Biomedical Sciences

University of Kalmar, Sweden

Lieve Nuytinck

Innogenetics NV

Ghent, Belgium
Michael K. Pangburn

Department of Biochemistry

University of Texas Health

Science Center

Tyler, Texas, USA

Ellinor I. B. Peerschke

Department of Pathology

Weill Medical College of Cornell

University

New York, New York, USA

József Prechl

Department of Immunology

Eötvös Loránd University

Pázmány P.s.1/C

Budapest, Hungary

Lavinia M. Proctor

Promics Ltd.

Brisbane, Queensland, Australia

Mariusz Z. Ratajczak

Stem Cell Biology Program

University of Louisville

Louisville, Kentucky, USA

Ryan Reca

Stem Cell Biology Program

University of Louisville

Louisville, Kentucky, USA

Robert M. Rennebohm

Columbus Children's Research Institute

Department of Pediatrics

The Ohio State University

Columbus, Ohio, USA

Daniel Rittirsch

Department of Traumatology, HandPlastic and Reconstructive Surgery

Ulm, Germany

Gunnar Ronquist

Department of Medical Sciences

Uppsala University Hospital, Sweden 
Ted M. Ross

Department of Medicine

University of Pittsburgh

Pittsburgh, Pennsylvania, USA

Brad H. Rovin

Department of Internal Medicine

The Ohio State University

Columbus, Ohio, USA

Horea Rus

Department of Neurology

University of Maryland

Baltimore, Maryland, USA

Marion Schneider

Department of Anesthesiology

University of Ulm Medical School

Ulm, Germany

Heike Schreiber

Department of Traumatology, Hand-

Plastic and Reconstructive Surgery

Ulm, Germany

Prakirti Sharma

School of Biomedical Sciences

University of Queensland, Australia

Ian A. Shiels

School of Biomedical Sciences

University of Queensland, Australia

Pei-an B. Shih

Department of Medicine

University of Pittsburgh School of Medicine

Pittsburgh, Pennsylvania, USA

Dinesh C. Soares

School of Chemistry/Institute of

Structural and Molecular Biology

University of Edinburgh

Edinburgh, UK

Jeong-Hyeon Sohn

Department of Ophthalmology

and Visual Science

University of Louisville

Louisville, Kentucky, USA
Tomonori Somamoto

Department of Bioscience and Biotechnology

Kyushu University

Fukuoka, Japan

Sergei A. Sosunov

Department of Neurological Surgery

Columbia University

College of Physicians \& Surgeons

New York, New York, USA

Edzard Spillner

Institut für Biochemie und

Lebensmittelchemie

Universität Hamburg, Germany

Michael E. Sughrue

Department of Neurological Surgery

Columbia University

College of Physicians \& Surgeons

New York, New York, USA

Lee Tantral

Department of Medicine

SUNY at Stony Brook

Stony Brook, New York, USA

Fletcher B. Taylor Jr

Oklahoma Medical Research

Foundation

Oklahoma City, Oklahoma, USA

Stephen M. Taylor

School of Biomedical Sciences

University of Queensland, Australia

Andrea J. Tenner

Departments of Molecular Biology and Biochemistry and Pathology

Center for Immunology

University of California

Irvine, California, USA

Nóra Terényi

Department of Immunology

Eötvös Loránd University

Budapest, Hungary 
Panagiotis A. Tsonis

Department of Biology

University of Dayton

Dayton, Ohio, USA

Martine Van Thielen

Innogenetics NV

Ghent, Belgium

Manfred Weiss

Department of Anesthesiology

University of Ulm Medical School

Ulm, Germany

Trent M. Woodruff

Promics Ltd.

Brisbane, Queensland, Australia

Yee-Ling Wu

Columbus Children's Research Institute

Integrated Biomedical Science

Graduate Program

The Ohio State University

Columbus, Ohio, USA
Marcin Wysoczynski

Stem Cell Biology Program

University of Louisville

Louisville, Kentucky, USA

Jun Yan

Stem Cell Biology Program

University of Louisville

Louisville, Kentucky, USA

Yan Yang

Columbus Children's Research Institute

Columbus, Ohio, USA

C. Yung Yu

Columbus Children's Research Institute

Department of Pediatrics

The Ohio State University

Columbus, Ohio, USA

Bi Zhou

Columbus Children's Research Institute

Columbus, Ohio, USA 